International Journal of Distributed and Parallel Systems (IJDPS) Vol.4, No.1, January 2013

\title{
A Performance evaluation of a Parallel BIOLOGICAL NETWORK MICROCIRCUIT IN NEURON
}

\author{
Salvatore Cuomo ${ }^{1}$, Pasquale De Michele ${ }^{2}$ and Francesco Piccialli ${ }^{3}$ \\ University of Naples "Federico II" Department of Mathematics and Applications, \\ Via Cinthia, 80126, Napoli, ITALY. \\ ${ }^{1}$ salvatore.cuomodunina.it \\ ${ }^{2}$ pasquale.demichele@unina.it \\ ${ }^{3}$ francesco.piccialli@gmail.com
}

\begin{abstract}
A critical issue in biological neural network modelling is the parameter tuning of a model by means ofthe numerical simulations to map a real scenario. This approach requires a huge amount ofcomputational resources to assesses the impact of every model value that, generally, changes thenetwork response. In this paper we analyse the performance of a CAlneural network microcircuit model for pattern recognition. Moreover, we investigate its scalability andbenefits on multicore and on parallel and distributed architectures.
\end{abstract}

\section{KEYWORDS}

Parallel Computing, GPU Computing, Performance Analysis, Programming, Biological Neural Network.

\section{INTRODUCTION}

Prototyping and developing computational codes of biological networks, in terms of reliability, efficient, and portable building blocks allow to the computational neuroscientists to simulate real cerebral behaviours and to validate theories and experiments. In prior work,we describedseveral mathematical models and software for biological cells modelling [13]; softwareare divided into two main categories: the general and the special purpose Problem Solving Environments (PSEs). The widely diffused PSEsareNEURON [8] and GENESIS [14]. These frameworks have large communities where the users collect and maintain databases of published computational models. In this work, we use NERUON to solve problems at several levels of biological details.

Many research papers ([9], [12], [15], [16] and [17])adopt parallel computing and scientific tools to increase the performance of novel neural network models. Generally, they highlight the biological results, without dealing on the computational aspects related to the parameter issue setting or to the simulation strategies.Moreover, they do not report detailed analysis of the parallel and distributed algorithms, which are essential to optimize a network in terms of its scalability and performance. In other words, building a neural network that reproduces a real scenario requires long and deep stepsto set the model parameters through numerical simulations. The validation of a computational model, by tuning different biological parameters, represents a critical issue in terms of memory allocations and computing time. For example, in [13] we have showed that the tuning of the external electrical stimuli and the random synapses of a modified CA1 model [17] requires a full computational time of $\mathrm{T}_{\text {tot }} \approx 9 \mathrm{~h}$ for a single cell. The adjustment phase increases the simulation timedramatically, when switching from a single cell to a DOI : $10.5121 /$ ijdps.2013.4102 
International Journal of Distributed and Parallel Systems (IJDPS) Vol.4, No.1, January 2013

network. In this work, the starting point is a multi-pattern modification of a CA1 neural network microcircuit model for pattern recognition [9], implemented in NEURON. In our experiments, the set-up of the network requires the storage of about 45GB of data and several days for any simulation. Moreover, the computational occupancy of the resources strongly depends on the model parameter variation. As a consequence, we study the scalability and the performance of the proposed modification, which is a crucial step to develop a network that recognizes patterns in a real scenario. This work is useful to highlight the strengths and weaknesses of the scientific and parallel computing strategies used in the implemented code. We prove that a pivotal role is played by the combined use of high performance computing techniques, including parallel scientific computing, multi-cores programming and GPU programming. Finally, we suggest solutions that may also be adopted to optimize the neural network code and to simulate a biological scenario in a more reliable time.

The paper in organised as follows. In the Section 2 we show our multi-pattern modification of the microcircuit. The Section 3 describes the numerical results and in the Section 4 performance considerations are discussed. Finally, in the Section 5 we report the conclusions.

\section{A Multi-Pattern Modification of a CA1 Microcircuit}

The model in [9] defines a neural network microcircuit model of the hippocampus, a brain region that is involved in the intermediate term storage of the memories that can be consciously recalled: the declarative memories $[1,2,3]$. The hippocampus contains two main types of cells: principal excitatory neurons that are the main information processors of this region, and a large kind of inhibitory inter-neurons that form connections locally [5, 6]. In [7] it is proposed an hippocampus feature, the theta rhythm $(4-7 \sim \mathrm{Hz})$, that contributes to the memory formation by separating encoding (storage) and retrieval (recall) of memories into different functional halfcycles. In particular, the model in [9] simulates in NEURON the firing time of different hippocampal cell types relative to the theta rhythm in anaesthetised animals, and addresses the roles played by the various types of inhibitory inter-neurons in the dynamical CA1 information processing.

Our modification consists of re-designing the model in [9] by introducing a new multi-pattern recognition strategy. Given a set of $N$ patterns, the model is able to store all the $N$ patterns with the Spike-timing-dependent plasticity (STDP) learning rule and subsequently recall these. STDP is a process that adjusts the strength of the connections between neurons in the brain. In STDP rule synapses increase (or decrease) their efficacy if the pre-synaptic spike arrives before (or after) the post-synaptic neuron is activated. For biological aims it is needed to investigate the CA1 network, by means of the recall stage of a large numbers of stored patterns. The complete knowledge of the neuronal phenomena requires the increasing of the patterns and multiple parametric simulations on the network.

As showed in the Figure 1, the model consists of 235 cells: 100 Pyramidal (P), 2 Basket (B), 1 BiStratified (BS), 1 Axo-Axonic (AA) and 1 OriensLacunosum-Moleculare (OLM), that are cells with biophysical properties adapted from literature; $100 \mathrm{CA} 3,20$ Entorinal Cortex (EC) and 10 medial SEPtum (SEP), that are cells whose behaviour is simulated by means of electrical stimuli. These cells are connected as showed in Table 1 . The duration of a single theta rhythm in the network is fixed to $250 \mathrm{~ms}: 125 \mathrm{~ms}$ for storage and $125 \mathrm{~ms}$ for recall phases. Repeating the theta cycles for a fixed number of times $(T)$ an alternation between phases of the storage and recall occurs. The algorithm is divided in two sub-algorithms: the storage and the recall. 
International Journal of Distributed and Parallel Systems (IJDPS) Vol.4, No.1, January 2013

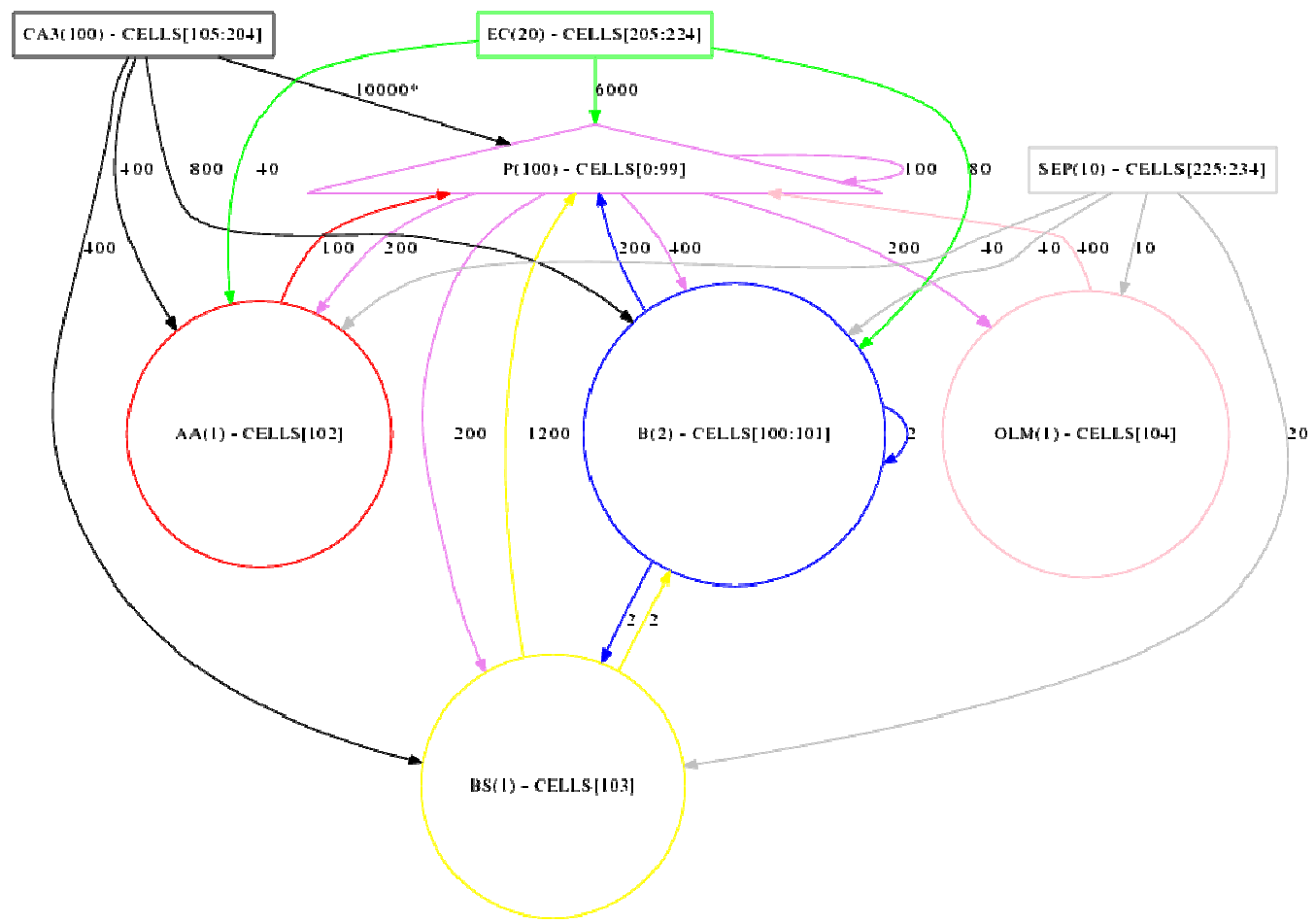

Figure 1. Network topology

\subsection{Storage Algorithm}

In the Figure 2 (on the left) the graphical representation of the multi-pattern Algorithm 1 is showed. This algorithm for the storage of $N$ patterns consists of many functionally dependent tasks.

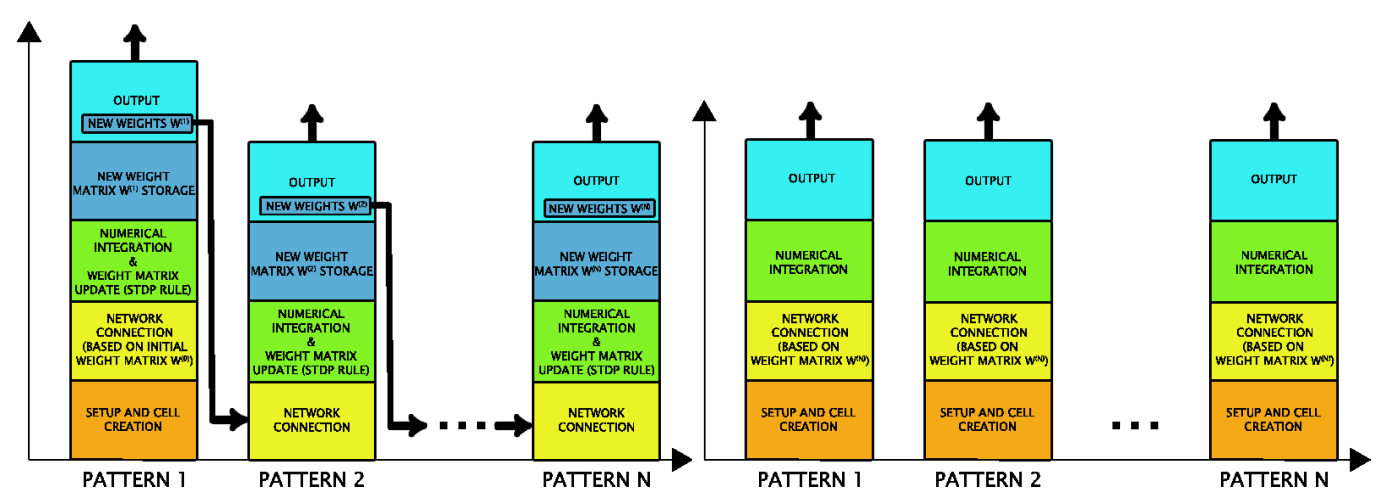

Figure 2. Graphical representations of the multi-pattern algorithms for the storage (on the left) and the recall (on the right) of $N$ patterns 
International Journal of Distributed and Parallel Systems (IJDPS) Vol.4, No.1, January 2013

Table 1. Network connections

\begin{tabular}{|c|c|c|c|c|c|}
\hline \multirow[b]{2}{*}{ Synapse } & \multirow[b]{2}{*}{$\begin{array}{c}\text { Number } \\
\text { of } \\
\text { sources }\end{array}$} & (a) & (b) & $(\mathrm{c})$ & \multirow[b]{2}{*}{$\begin{array}{c}\text { Number of synapses } \\
\text { in total }= \\
((\mathbf{a}) \times(\mathbf{b})) \times(\mathbf{c})\end{array}$} \\
\hline & & $\begin{array}{c}\text { Number } \\
\text { of targets }\end{array}$ & $\begin{array}{l}\text { Number of } \\
\text { connections }\end{array}$ & $\begin{array}{c}\text { Number of } \\
\text { synapses in a } \\
\text { single } \\
\text { connection }\end{array}$ & \\
\hline $\mathrm{EC} \rightarrow \mathrm{B}$ & 20 & 2 & 20 & 2 & $(2 \times 20) \times 2=80$ \\
\hline $\mathrm{EC} \rightarrow \mathrm{AA}$ & 20 & 1 & 20 & 2 & $(1 \times 20) \times 2=40$ \\
\hline $\mathrm{EC} \rightarrow \mathrm{P}$ & 20 & 100 & 20 & 3 & $(100 \times 20) \times 3=6000$ \\
\hline $\mathrm{CA} 3 \rightarrow \mathrm{B}$ & 100 & 2 & 100 & 4 & $(2 \times 100) \times 4=800$ \\
\hline $\mathrm{CA} 3 \rightarrow \mathrm{AA}$ & 100 & 1 & 100 & 4 & $(1 \times 100) \times 4=400$ \\
\hline $\mathrm{CA} 3 \rightarrow \mathrm{BS}$ & 100 & 1 & 100 & 4 & $(1 \times 100) \times 4=400$ \\
\hline $\mathrm{CA} 3 \rightarrow \mathrm{P}$ & 100 & 100 & 100 & 1 & $(100 \times 100) \times 1=10000$ \\
\hline $\mathrm{SEP} \rightarrow \mathrm{B}$ & 10 & 2 & 10 & 2 & $(2 \times 10) \times 2=40$ \\
\hline $\mathrm{SEP} \rightarrow \mathrm{AA}$ & 10 & 1 & 10 & 2 & $(1 \times 10) \times 2=20$ \\
\hline $\mathrm{SEP} \rightarrow \mathrm{BS}$ & 10 & 1 & 10 & 2 & $(1 \times 10) \times 2=20$ \\
\hline $\mathrm{SEP} \rightarrow \mathrm{OLM}$ & 10 & 1 & 10 & 1 & $(1 \times 10) \times 1=10$ \\
\hline $\mathrm{P} \rightarrow \mathrm{P}$ & 100 & 100 & 1 & 1 & $(100 \times 1) \times 1=100$ \\
\hline $\mathrm{P} \rightarrow \mathrm{B}$ & 100 & 2 & 100 & 2 & $(2 \times 100) \times 2=400$ \\
\hline $\mathrm{P} \rightarrow \mathrm{AA}$ & 100 & 1 & 100 & 2 & $(1 \times 100) \times 2=200$ \\
\hline $\mathrm{P} \rightarrow \mathrm{BS}$ & 100 & 1 & 100 & 2 & $(1 \times 100) \times 2=200$ \\
\hline $\mathrm{P} \rightarrow \mathrm{OLM}$ & 100 & 1 & 100 & 2 & $(1 \times 100) \times 2=200$ \\
\hline $\mathrm{B} \rightarrow \mathrm{P}$ & 2 & 100 & 2 & 1 & $(100 \times 2) \times 1=200$ \\
\hline $\mathrm{B} \rightarrow \mathrm{B}$ & 2 & 2 & 1 & 1 & $(2 \times 1) \times 1=2$ \\
\hline $\mathrm{B} \rightarrow \mathrm{BS}$ & 2 & 1 & 2 & 1 & $(1 \times 2) \times 1=2$ \\
\hline $\mathrm{AA} \rightarrow \mathrm{P}$ & 1 & 100 & 1 & 1 & $(100 \times 1) \times 1=100$ \\
\hline $\mathrm{BS} \rightarrow \mathrm{P}$ & 1 & 100 & 1 & 12 & $(100 \times 1) \times 12=1200$ \\
\hline $\mathrm{BS} \rightarrow \mathrm{B}$ & 1 & 2 & 1 & 1 & $(2 \times 1) \times 1=2$ \\
\hline $\mathrm{OLM} \rightarrow \mathrm{P}$ & 1 & 100 & 1 & 4 & $(100 \times 1) \times 4=400$ \\
\hline & & $\begin{array}{l}\text { itialize } W^{(0)} \\
\text { ETUP AND } \\
\mathbf{r} i=1 \text { to } N \\
\text { NETWORK } \\
\text { NUMERICA } \\
\text { WEIGHT M } \\
\text { NEW WEIG } \\
\text { OUTPUT } \\
\text { ad for }\end{array}$ & $\begin{array}{l}\text { ELL CREATIOI } \\
\text { lo } \\
\text { SONNECTION } \\
\text { INTEGRATIO } \\
\text { TRIX } W^{(i)} \text { UP } \\
\text { IT MATRIX } W^{(}\end{array}$ & $\begin{array}{l}\text { ased on } W^{(i-1)} \\
\text { and } \\
\text { ATE with STDP r } \\
\text { STORAGE }\end{array}$ & \\
\hline
\end{tabular}

Algorithm 1. Multi-patterns storage algorithm

The tasks of a single pattern are organized in stacks, with different execution steps. In detail, these steps are: 1) the network initializing (for the first pattern only); 2) the setup of thenetwork connections by means of a suitable weight matrix W, computed with STDP rule; 3) the numerical integration and the Wupdate; 4) the storage of $\mathbf{W}$; 5) the output generation. The numerical integration consists of the solutions of systems of Ordinary Differential Equations (ODEs) that models the cell computational behaviour. A detailed description of the model is in the Appendix. 
International Journal of Distributed and Parallel Systems (IJDPS) Vol.4, No.1, January 2013

The $\mathbf{W}$ matrix covers a key role in the adaptive multi-pattern recognition, because it is used to opportunely calibrate the network connections, taking into account the synaptic plasticity. In particular, for each pattern $i(1 \leq i \leq N)$, the model returns:

$$
\mathbf{W}^{(i)}=s\left(\mathbf{W}^{(i-1)}, \mathbf{c}^{(i)}, \mathbf{p}^{(i)}\right)
$$

where $\mathbf{W}^{(i-1)}$ is the weight matrix obtained by the $(i-1)$-th pattern storage $\left(\mathbf{W}^{(0)}=0\right), \mathbf{c}^{(\mathrm{i})}$ is a network connection vector, $\mathbf{p}^{(\mathrm{i})}$ is the $i$-th pattern to be stored, and $s$ is a function that represents the STDP rule.

The stacks of the storage algorithm are functionally dependent, and our implementation strategy consists of parallelizing the network activity on a single stack (pattern). By using a round-robin strategy, the network cells are distributed among the available hosts [10], as showed in Figure 3.

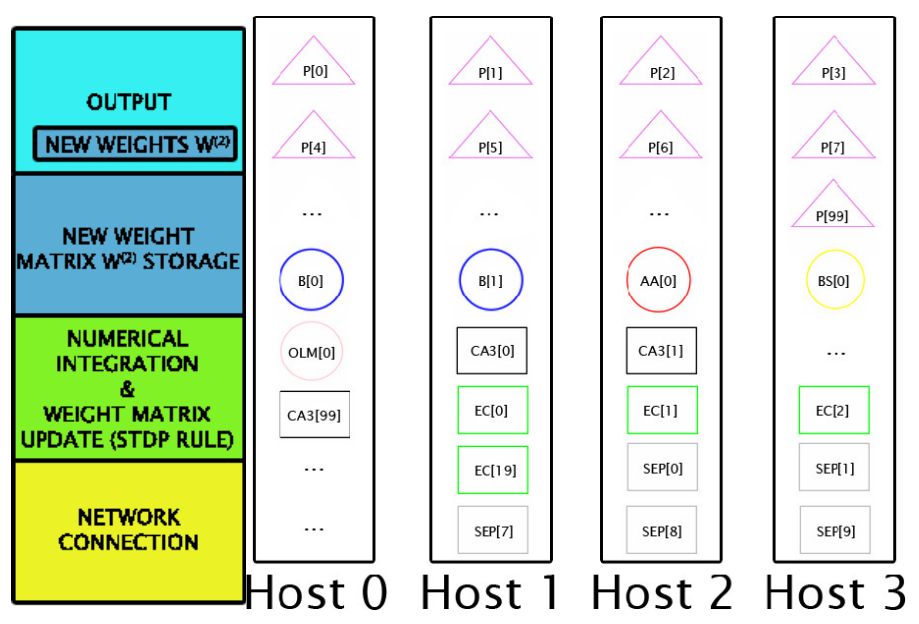

Figure 3. Example of parallelization with 4 available hosts, for a generic execution stack 
International Journal of Distributed and Parallel Systems (IJDPS) Vol.4, No.1, January 2013

From parallel computing point of view, the strategy is the following:

(1) a processor integrates the computational modelon its assigned group of cells;

(2) if a spike event occurs between source and target cells, a network communication (NetCon)is activated;

(3) theweight matrix information are collected in parallel with a "compare and exchange" strategy.

The parallel performance of the storage algorithm will be discussed in the Section 3 .

\subsection{Recall Algorithm}

In Figure 2 (on the right), the graphical representation of the recall Algorithm 2 is showed.At the $i$-th pattern, the model returns:

$$
O U T^{(i)}=r\left(\mathbf{W}^{(N)}, \mathbf{c}^{(i)}, \mathbf{p}^{(i)}\right)
$$

where $\mathbf{W}^{(N)}$ is the weight matrix obtained from the previously storage of the $N$ patterns, $\mathbf{c}^{(i)}$ is a network connection vector, $\mathbf{p}^{(i)}$ is the pattern to be recalled, $r$ is a function that evaluates the recall, $O U T^{(i)}$ is the biological output related on recall performance.

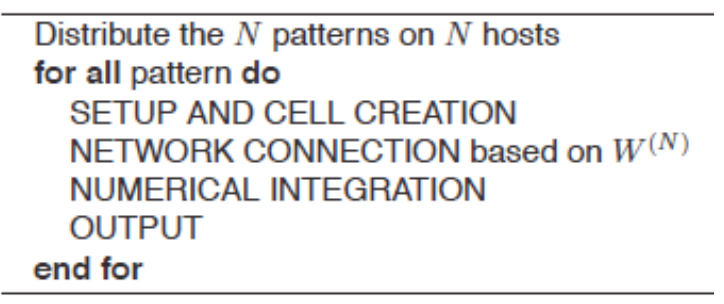

Algorithm 2. Multi-patterns recall algorithm

During the recall of the $N$ patterns, all stacks are independent, thus it is possible both to distribute and parallelize the execution of these. In detail, a single pattern, that will be recalled, is parallelized in the same way of the storage phase. Let be $K=N * M$ the number of the available hosts on the parallel and distributed architecture,in a first phase $N$ master hosts are selected in order to recall a stored pattern. In a second phase each master host sets the network on $M$ assigned hosts and carries out the recall algorithm, i.e. it executes a stack. For example, suppose we have to recall 10 patterns on a parallel architecture with 8 core hosts. Each host recalls a stored pattern and executes the stack on its 8 core blade.

In Figure 4, the output of the microcircuit for one pattern of the recall algorithm is showed. The simulation is carried out on $6100 \mathrm{~ms}$ with 24 theta cycles and shows the input spikes, the recall quality, the number of spikes of pyramidal cell and the pyramidal cells involved in the synaptic activity. After a phase of network setup and calibration, we point out that the matrix of the weight $\mathbf{W}$ obtained by the storage algorithm, is used to recall a fixed memory pattern with the quality showed in Figure 4. 

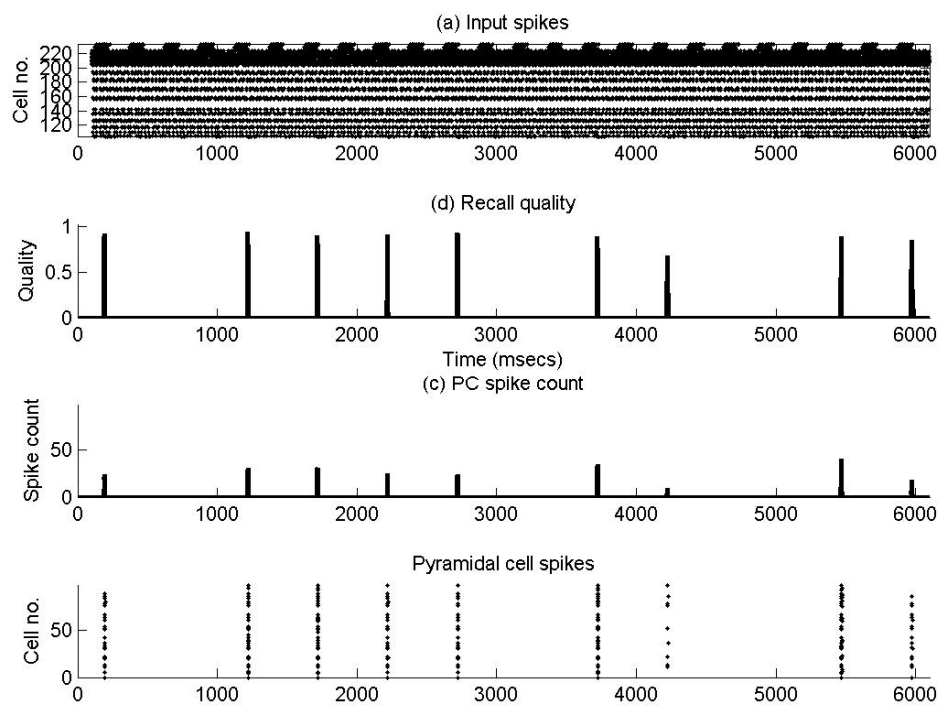

Figure 4. Biological output carried out after a pattern recall during $6100 \mathrm{~ms}$

In this work we do not discuss about the biological results of the computational neural network, but we remark that a simulation on a workstation with 8 cores requires, at this time, $\sim 910$ seconds. This experiment is reported with the aim to show that it is needed to repeat the simulations by tuning the network parameters. Using the network with a large number of patterns and theta cycles, it is a preliminary step for validating the CA1 microcircuit from a biological standpoint. In the next Section we discuss the computational performance of these algorithms.

\section{Multi-core and Parallel Distributed context COMParisons}

The model is implemented by using the problem solving scientific environment NEURON (v.7.1). The performance tests were carried out on the S.Co.P.E. Grid Infrastructure of University of Naples "Federico II", that consists of 300 blade servers (nodes), each of which with 8 cores "Intel Xeon E5410" at $2.33 \mathrm{GHz}$ (2400 cores in total), connected with several kinds of links: Infiniband, Fiber Channel, $1 \mathrm{~Gb}$ and $10 \mathrm{~Gb}$ Ethernet links. Storage and recall algorithms are performed on a set of 10 patterns, both characterized by duration of each thetacycle of $250 \mathrm{~ms}$ and an initial delay of $100 \mathrm{~ms}$. The storage is performed on two different architectures: multicore and distributed.

In order to evaluate the storage and recall performance on these architectures, different parameters are taken into account. The Runtime is the total execution time for the application; Wait is the time spent for exchanging spikes during a simulation; Step is the time spent to integrate ODE systems, checking thresholds, and delivering events (it corresponds to the amount of time needed to numerical integration and weight matrix $\mathbf{W}$ updating); Setup and cell creation is the time to setupbiological parameters and to create all cells of the network; Network connection is the time to setup network connection; New weight matrix storage is the time to collect, from each host, the values of the weight matrix and storing these for the next pattern to be processed; Output is the time to store biological output information; Others is the time to collect non-functional information. 


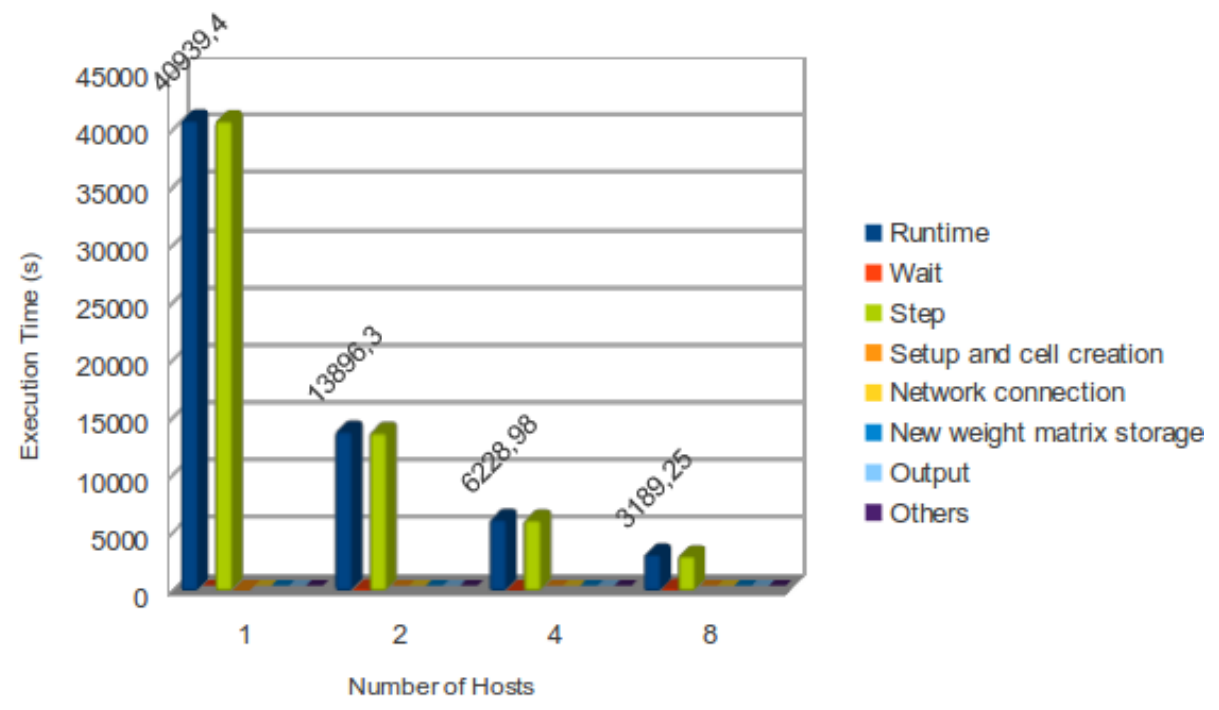

Figure 5. Execution times for the storage of 10 patterns with 8 theta cycles on a multicore architecture

The Figure 5 shows that for the storage phase, on a multicore architecture, increasing the number of hosts (from 1 to 8 ) leads to a huge reduction of Runtime, substantially equivalent to the Step time. In fact, from the Figure 6, it is possible to see a reduction ofStep from the 100\% to the $95,47 \%$ of the Runtime, moving from 1 to 8 hosts. Hence, the other execution steps (setup and cell creation, network connection, weight matrix storage and output generation) do not affect the overall time.

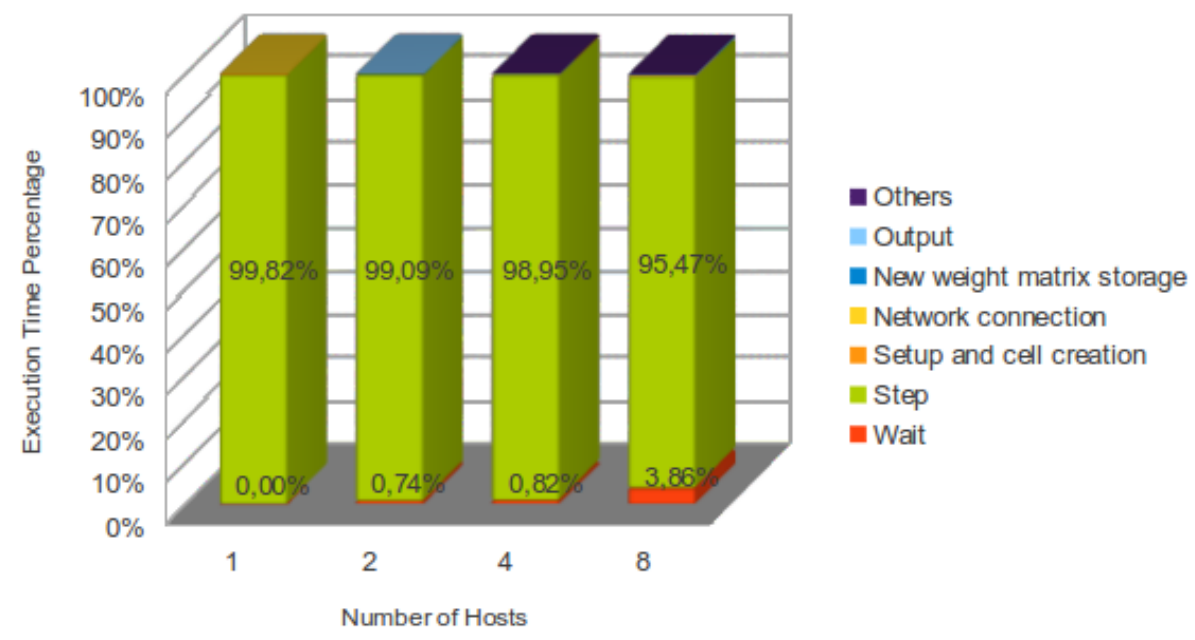

Figure 6. Runtime percentages for the storage of 10 patterns with 8 theta cycles on a multicore architecture

Moreover, the Figure 6 shows a slight increment of the Wait time (from $0 \%$ to 3,86\% of the Runtime) due to the growing number of communications among the cells mapped on different hosts. This phenomenon is amplified when moving from a multicore to a distributed architecture. 


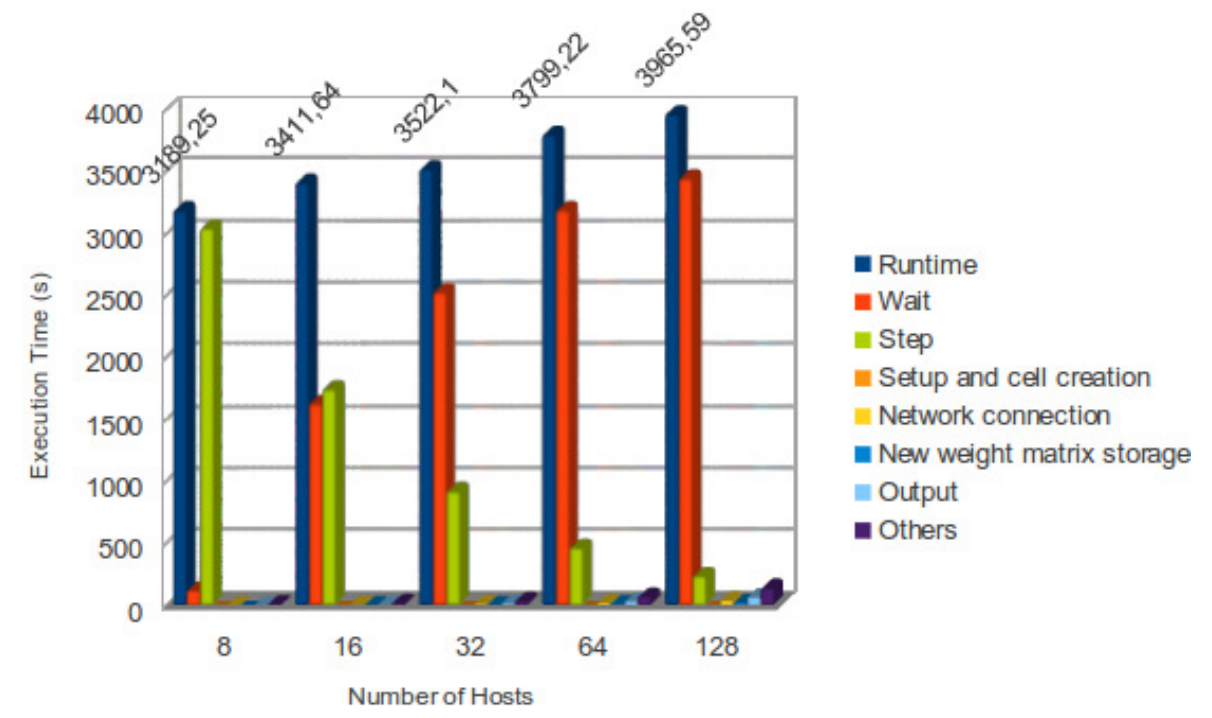

Figure 7. Execution times for the storage of 10 patterns with 8 theta cycles on a distributed architecture

The Figure 7 shows that on a distributed architecture a slight worsening of the performance occurs. In detail, the time needed for the numerical integration (Step) continues to decrease with doubling of the host number and there is a strong increment of the Waittime (the amount of time needed to synchronize the communication between two cells mapped on two different hosts). The Figure 8 confirms this: with 16 hosts (distributed on 2 different nodes) Wait time reaches the $47,76 \%$ of the Runtime, while with 128 hosts it further increases, reaching the $86,93 \%$ of the Runtime.

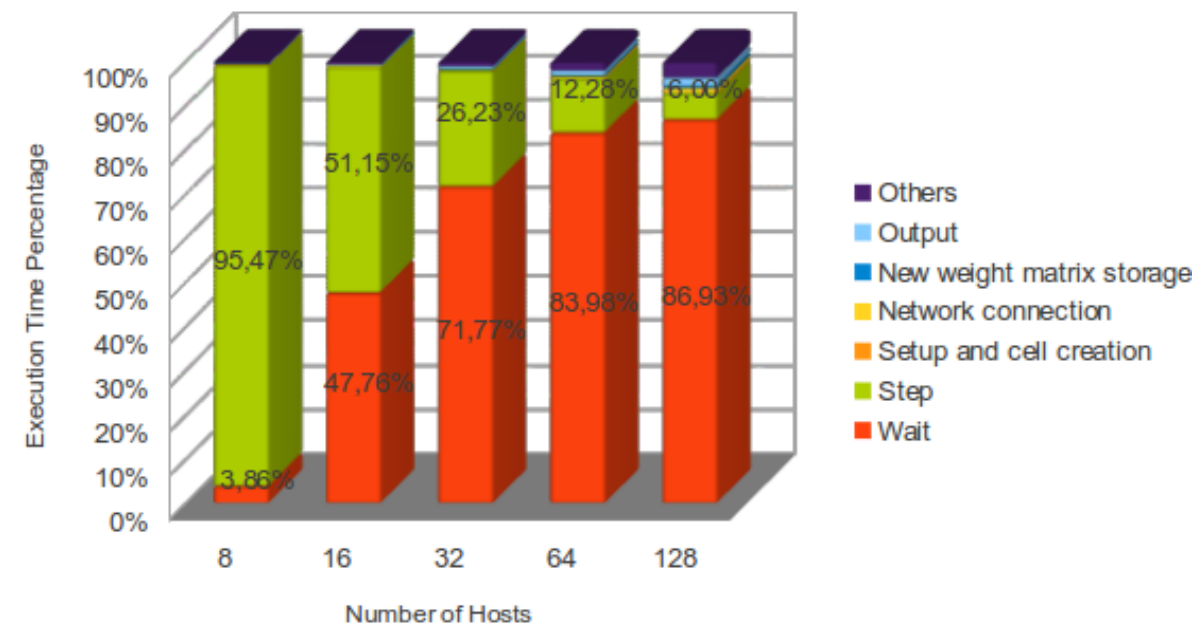

Figure 8. Runtime percentages for the storage of 10 patterns with 8 theta cycles on a distributed architecture

Moreover, this behaviour is particularly clear by observing the Figure 9. NEURON uses an event delivery system to implement spike-triggered synaptic transmission between cells. Detection of a spike launches an event that, after an appropriate delay, will be delivered to a 
International Journal of Distributed and Parallel Systems (IJDPS) Vol.4, No.1, January 2013

target cell. The basic problem that has to be overcome in a parallel simulation environment is that the source cell and its target usually do not exist on the same host [10]. Hence, it is evident that, moving from a multicore to a distributed architecture, the time for synchronize different hosts rises with increasing of the involved host number.

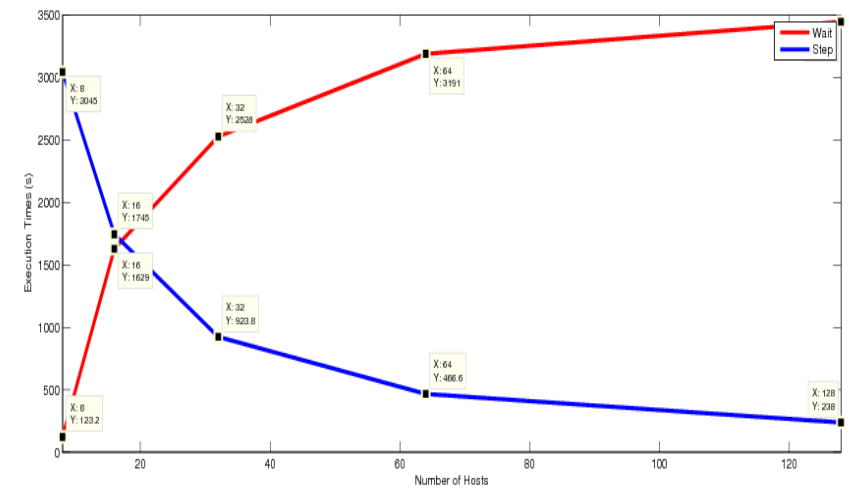

Figure 9. Step and Wait curves with the increasing of number of hosts

On a multicore architecture, the recall algorithm has the same performance we have discussed for the storage phase. On the other hand, as we mentioned earlier, it is possible to implement the recall algorithm on a distributed architecture: in this way, each algorithm performs the recall of a single pattern on a node of the architecture. A typical parametric execution of a distributed recall phase is characterized by the pair (Number of pattern, Number of theta cycles). In the Table 2 the performance of the parametric execution (10 patterns, 16 theta cycles) are showed. The recall of $N$ patterns is performed in $\sim 600 s$, that is the time needed to recall a single pattern only, obtaining a substantial performance improvement: without the distribution of the algorithm, the Runtime would have been equal to $\sim 6000$ s.

Table 2. Execution times for the recall of 10 patterns with 16 theta cycles, distributing the model

\begin{tabular}{|c|c|c|c|c|c|c|}
\hline Pattern ID & Runtime & Wait & Step & \#Spikes & $\begin{array}{c}\text { Set and } \\
\text { Connect }\end{array}$ & Output \\
\hline 1 & 608,775 & 8,71033 & 597,987 & 7975 & 0,54125 & 0,61625 \\
\hline 2 & 608,861 & 8,93715 & 598,457 & 8149 & 0,54125 & 0,6375 \\
\hline 3 & 607,072 & 8,51312 & 597,083 & 8061 & 0,54125 & 0,64875 \\
\hline 4 & 607,812 & 8,67061 & 597,683 & 8033 & 0,54125 & 0,635 \\
\hline 5 & 607,119 & 9,37756 & 596,286 & 7946 & 0,54125 & 0,6275 \\
\hline 6 & 607,035 & 8,71243 & 596,895 & 7992 & 0,54125 & 0,6325 \\
\hline 7 & 607,726 & 8,79549 & 597,473 & 8030 & 0,54125 & 0,62125 \\
\hline 8 & 607,191 & 8,39568 & 597,321 & 7848 & 0,54125 & 0,63875 \\
\hline 9 & 608,27 & 10,0982 & 596,692 & 8022 & 0,54125 & 0,63625 \\
\hline 10 & 608,42 & 9,3668 & 597,576 & 8046 & 0,54125 & 0,6325 \\
\hline
\end{tabular}


International Journal of Distributed and Parallel Systems (IJDPS) Vol.4, No.1, January 2013

computing and exporting the code of our microcircuit on a more general-purpose simulator that supports the GPU.

\section{ACKNOWLEDGEMENTS}

This paper is financial supported by Multicentre Research Project funded by Compagnia di San Paolo: "Molecular Mechanisms of Memory: Identification and Modelling".

\section{A. APPENDix: Mathematical Formalism}

\section{A.1. Pyramidal Cells}

The somatic (s), axonic (a) and radiatum (rad), lacunosum-moleculare (LM) and oriens (ori) dendritic compartments obey the following current balance equations:

$$
\begin{aligned}
& C \frac{d V_{s}}{d t}=-I_{L}-I_{N a}-I_{k d r}-I_{A}-I_{M}-I_{h}-I_{s A H P}-I_{m A H P}-I_{C a L}-I_{C a T}-I_{C a R}-I_{b u f f}-I_{s y n} \\
& C \frac{d V_{a}}{d t}=-I_{L}-I_{N a}-I_{k d r}-I_{M}-I_{s y n} \\
& C \frac{d V_{\text {rad, ori }}}{d t}=-I_{L}-I_{N a}-I_{k d r}-I_{A}-I_{M}-I_{h}-I_{s A H P}-I_{m A H P}-I_{C a L}-I_{C a T}-I_{C a R}-I_{b u f f}-I_{s y n} \\
& C \frac{d V_{L M}}{d t}=-I_{L}-I_{N a}-I_{k d r}-I_{A}-I_{s y n}
\end{aligned}
$$

where $I_{L}$ is the leak current, $I_{N a}$ is the fast sodium current, $I_{k d r}$ is the delayed rectifier potassium current, $I_{A}$ is the A-type $K^{+}$current, $I_{M}$ is the M-type $K^{+}$current, $I_{h}$ is a hyperpolarizing h-type current, $I_{C a L}, I_{C a T}$ and $\mathrm{I}_{\mathrm{CaR}}$ are the L-, T- and R-type $\mathrm{Ca}^{2+}$ currents, respectively, $I_{s A H P}$ and $I_{m A H P}$ are slow and medium $\mathrm{Ca}^{2+}$ activated $\mathrm{K}^{+}$currents, $I_{b u f f}$ is a calcium pump/buffering mechanism and $I_{s y n}$ is the synaptic current.

The sodium current is described by:

$$
I_{N a}=\bar{g}_{N a} \times m^{2} \times h \times s \times\left(V-E_{N a}\right)
$$

The delayed rectifier current is given by:

$$
I_{K d r}=\bar{g}_{K d r} \times m^{2} \times\left(V-E_{K}\right)
$$

The fast inactivating A-type $K^{+}$current is described by:

$$
I_{A}=\bar{g}_{A} \times \bar{n}_{A} \times\left(V-E_{K}\right)
$$

The hyperpolarizing h-current is given by:

$$
I_{h}=g_{h} \times t t \times\left(V-E_{h}\right)
$$

The slowly activating voltage-dependent potassium current, $I_{M}$, is given by the equation:

$$
I_{m}=10^{-4} \times T_{a d j}\left({ }^{\circ} C\right) \times \bar{g}_{m} \times m \times\left(V-E_{K}\right)
$$

The slow after-hyperpolarizing current, $I_{S A H P}$, is given by:

$$
I_{s A H P}=\bar{g}_{s A H P} \times m^{3} \times\left(V-E_{K}\right)
$$


International Journal of Distributed and Parallel Systems (IJDPS) Vol.4, No.1, January 2013

The medium after-hyperpolarizing current, $\mathrm{I}_{\mathrm{mAHP}}$, is given by:

$$
I_{m A H P}=\bar{g}_{m A H P} \times m \times\left(V-E_{K}\right)
$$

The somatic high-voltage activated (HVA) L-type $\mathrm{Ca}^{2+}$ current is given by:

$$
I_{C a L}^{S}=\bar{g}_{C a L}^{-} \times m \times \frac{0.001 m M}{0.001 m M+c a_{\text {in }}} \times g h k\left(V, c a_{\text {in }}, c a_{\text {out }}\right)
$$

where the dendritic L-type calcium channels have different kinetics:

$$
I_{C a L}^{d}=\bar{g}_{C a L}^{d} \times m^{3} \times h \times\left(V-E_{C a}\right)
$$

The low-voltage activated (LVA) T-type $C \mathrm{a}^{2+}$ channel kinetics are given by:

$$
I_{C a T}=\bar{g}_{C a T} \times m^{2} \times h \times \frac{0.001 m M}{0.001 m M+c a_{i n}} \times g h k\left(V, c a_{i n}, c a_{\text {out }}\right)
$$

The HVA R-type $\mathrm{Ca}^{2+}$ current is described by:

$$
I_{C a R}=\bar{g}_{C a R} \times m^{3} \times h \times\left(V-E_{C a}\right)
$$

Finally, a calcium pump/buffering mechanism is inserted at the cell body and along the apical and basal trunk. The factor for $\mathrm{Ca}^{2+}$ entry was changed from $f_{e}=10000$ to $\mathrm{f}_{e}=10000 / 18$ and the rate of calcium removal was made seven times faster.

The kinetic equations are given by:

$$
\begin{gathered}
\text { drive_channel }=\left\{\begin{array}{cc}
-f^{e} \times \frac{I_{C a}}{0.2 \times F A R A D A Y} & \text { if drive_channel }>0 \mathrm{mM} / \mathrm{ms} \\
0 & \text { otherwise }
\end{array}\right. \\
\frac{d c a}{d t}=\text { drive_channel }+\frac{\left(10^{-4}(\mathrm{mM})-\mathrm{ca}\right)}{7 \times 200(\mathrm{~ms})} .
\end{gathered}
$$

\section{A.2. Axo-axonic, Basket and Bistratified Cells}

All compartments obey the following current balance equation:

$$
C \frac{d V}{d t}=-I_{e x t}-I_{L}-I_{N a}-I_{K d r, f a s t}-I_{A}-I_{C a L}-I_{C a N}-I_{A H P}-I_{C}-I_{s y n}
$$

where $C$ is the membrane capacitance, $V$ is the membrane potential, $I_{L}$ is the leak current, $I_{N a}$ is the sodium current, $I_{K d r \text { fast }}$ is the fast delayed rectifier $K^{+}$current, $I_{A}$ is the A-type $K^{+}$current, $I_{C a L}$ is the L-type $\mathrm{Ca}^{2+}$ current, $I_{C a N}$ is the N-type $\mathrm{Ca}^{2+}$ current, $I_{A H P}$ is the $\mathrm{Ca}^{2+}$-dependent $\mathrm{K}^{+}$ $(S K)$ current, $I_{C}$ is the $C a^{2+}$ and voltage-dependent $K^{+}(B K)$ current and $I_{s y n}$ is the synaptic current.

The sodium current is described by:

$$
I_{N a}=g_{N a} m^{3} h\left(V-E_{N a}\right)
$$

The fast delayed rectifier $K^{+}$current, $I_{K d r \text {,fast }}$ is given by:

$$
I_{K d r, f a s t}=g_{K d r, f a s t} n_{f}^{4}\left(V-E_{K}\right)
$$


The N-type $\mathrm{Ca}^{2+}$ current, $I_{C a N}$, is given by:

$$
I_{C a N}=g_{C a N} c^{2} d\left(V-E_{C a}\right)
$$

The $C a^{2+}$-dependent $K^{+}(S K)$ current, $I_{A H P}$, is described by:

$$
I_{A H P}=g_{A H P} q^{2}\left(V-E_{K}\right)
$$

The A-type $K^{+}$current, $I_{A}$, is described by

$$
I_{A}=g_{A} a b\left(V-E_{K}\right)
$$

The L-type $\mathrm{Ca}^{2+}$ current, $I_{C a L}$, is described by:

$$
I_{C a L}=g_{C a L} \times s_{\infty}^{2} \times V \times \frac{1-\frac{\left[C a^{2+}\right]_{i}}{\left[C a^{2+}\right]_{0}} e^{2 F V / k T}}{1-e^{2 F V / k T}}
$$

where $g_{C a L}$ is the maximal conductance, $s_{\infty}$ is the steady-state activation variable, $F$ is Faraday's constant, $T$ is the temperature, $k$ is Boltzmann's constant, $\left[\mathrm{Ca}^{2+}\right]_{0}$ is the equilibrium calcium concentration and $\left[\mathrm{Ca}^{2+}\right]_{i}$ is described in Eq (25).

\section{A.3. OLM Cell}

The somatic (s), axonic (a) and dendritic (d) compartments of each OLM cell obeyed the following current balance equations:

$$
\begin{gathered}
C \frac{d V_{s}}{d t}=-I_{e x t}-I_{L}-I_{N a, s}-I_{K, s}-I_{A}-I_{h}-I_{s y n} \\
C \frac{d V_{d}}{d t}=-I_{e x t}-I_{L}-I_{N a, d}-I_{K, d}-I_{A}-I_{s y n} \\
C \frac{d V_{a}}{d t}=-I_{e x t}-I_{L}-I_{N a, d}-I_{K, d}
\end{gathered}
$$

The sodium current is described by:

$$
I_{N a}=g_{N a} m^{3} h\left(V-E_{N a}\right)
$$

where $m$ and $h$ are the activation and inactivation variables, respectively.

The potassium current, $I_{K}$, is described by:

$$
I_{K}=g_{K} n^{4}\left(V-E_{K}\right)
$$

wheren is the activation variable for this channel.

The transient potassium current, $I_{A}$, is described by:

$$
I_{A}=g_{A} a b\left(V-E_{K}\right)
$$

where $a$ and $b$ are the activation and inactivation variables, respectively.

The nonspecific cation channel, $I_{h}$, is described by:

$$
I_{h}=g_{h} r\left(V-E_{r}\right)
$$


International Journal of Distributed and Parallel Systems (IJDPS) Vol.4, No.1, January 2013

where $r$ is the activation variable for this channel.

\section{A.4. Septal Cells}

Septal cell output was modeled as bursts of action potentials using a presynaptic spike generator. A spike train consisted of bursts of action potentials at a mean frequency of $50 \mathrm{~Hz}$ for a half-u cycle ( $125 \mathrm{~ms}$; corresponding to a recall period) followed by a half-u cycle of silence. Due to $40 \%$ noise in the interspike intervals, the 10 spike trains in the septal population were asynchronous.

\section{A.5. Entorinal Cortical Cells}

EC cells were also modeled as noisy spike trains, using a pre-synaptic spike generator. A spike train consisted of spikes at an average gamma frequency of $40 \mathrm{~Hz}$, but with individual spike times Gaussian-distributed around the regular ISI of $25 \mathrm{~ms}$, with a standard deviation of 0.2. The population of EC inputs fired asynchronously.

\section{A.6. CA3 Pyramidal Cells}

CA3 pyramidal cells were modeled as spike trains of the same form and with the same characteristics (mean frequency and noise level) as the EC cells. Onset of CA3 firing was delayed by $9 \mathrm{~ms}$ relative to the EC trains to model the respective conduction delays of direct and trisynaptic loop inputs to CA1.

\section{REFERENCES}

[1] Eichenbaum, H., Dunchenko, P., Wood, E., Shapiro, M. and Tanila, H. (1999). The hippocampus, memory and place cells: Is it spatial memory or a memory of space? NEURON, 23, 209-226.

[2] Andersen, P., Morris, R., Amaral, D., Bliss, T. and O'Keede, J. (2007).The Hippocampus Book. Oxford: University Press.

[3] Wood, E., Dunchenko, P. and Eichenbaum, H. (1999). The global record of memory in hippocampal neuronal activity.NATURE, 397, 613-616.

[4] Treves, A. and Rolls, E. (1994). Computational analysis of the role of the hippocampus in memory.HIPPOCAMPUS, 4, 374-391.

[5] Freund, T.F. and Buzsaki, G. (1996). Interneurons of the hippocampus.HIPPOCAMPUS, 6, $347-$ 470.

[6] Somogyi, P. and Klausberger, T. (2005). Defined types of cortical interneurons structure space and spike timing in the hippocampus. J PHYSIOL, 562, 9-26.

[7] Hasselmo, M.E., Bodelon, C. and Wyble, B. (2002). A proposed function of the hippocampal theta rhythm: Separate phases of encoding and retrieval of prior learning. NEURAL COMPUT, 14, 793-817.

[8] Carnevale, N.T. and Hines, M.L. (2006). The NEURON book.Cambridge University Press.

[9] Cutsuridis, V., Cobb, S. and Graham, B.P. (2010).Encoding and Retrieval in a Model of the Hippocampal CA1 Microcircuit.HIPPOCAMPUS, 20, 423-446.

[10] Hines, M.L. and Carnevale, N.T. (2008). Translating network models to parallel hardware in NEURON. J NEUROSCI METH, 169(2), 425-455.

[11] Richert, M., Nageswaran, J.M., Dutt N. and Krichmar, J.L. (2011).An efficient simulation environment for modeling large-scale cortical processing. FRONT NEUROINFORM, 5:(19), doi: 10.3389/fninf.2011.00019. 
International Journal of Distributed and Parallel Systems (IJDPS) Vol.4, No.1, January 2013

[12] Myers, C.E. and Scharfman, H.E. (2011). Pattern Separation in the Dentate Gyrus: A Role for the CA3 Backprojection, HIPPOCAMPUS, 21:1190-1215.

[13] Cuomo, S., De Michele, P. and Chinnici, M. (2011). Parallel tools and techniques for biological cells modelling, BuletinulInstitutuluiPolitehnic DIN IASI, Automatic Control and Computer Science Section, LVII (LXI), Fasc. 3, 2011, pp. 61-75, ISSN 1200-2169.

[14] Bower, J.M., Beeman, D. et al. (2003). The Book of Genesis. Internet Edition.

[15] Cutsuridis, V., Hunter, R., Cobb, S. and Graham, B.P. (2007). Storage and recall in the CA1 microcircuit of the hippocampus: A biophysical model. Vol. 8(suppl 2), 16th Annual Computational Neuroscience Meeting CNS*2007, Toronto, Canada, BMC NEUROSCI, p. 33.

[16] Cutsuridis, V. and Wenneckers, T. (2009).Hippocampus, microcircuits and associative memory. HIPPOCAMPUS 22, 1120-1128.

[17] Bianchi, D., Marasco, A., Limongiello, A., Marchetti, C., Marie, H., Tirozzi, B. and Migliore, M. (2011). On the mechanisms underlying the depolarization block in the spiking dynamics of CA1 pyramidal neurons. J COMPUT NEUROSCI - DOI 10.1007/s10827-012-0383-y.

[18] Izhikevich, E.M. (2004). Which model to use for cortical spiking neurons? IEEE TRANS NEURAL NETW. 15, 1063-1070.

\section{Authors}

Salvatore Cuomo is an Assistant Professor at University of Naples "Federico II".

Pasquale De Michele is a Ph.D.Student in "Computer Science" at University of Naples "Federico II".

Francesco Piccialli is a Graduated in "Computer Science" at University of Naples "Federico II". 